\title{
Cerebrovascular Segmentation Based on Hidden Markov Model in Phase-Contrast Angiography Images
}

\section{Kangneng Zhou}

University of Science and Technology Beijing

\section{Cheng Chen}

University of Science and Technology Beijing

\section{Yuanyuan Lu}

Chinese PLA General Hospital

\section{Xinmeng Guo}

University of Science and Technology Beijing

Wubin Li

University of Science and Technology Beijing

\section{Huilin Wang}

University of Science and Technology Beijing

Xintong Wu

University of Science and Technology Beijing

Ruoxiu Xiao ( $\sim$ xiaoruoxiu@ustb.edu.cn)

University of Science and Technology Beijing https://orcid.org/0000-0002-2721-4813

\section{Research}

Keywords: Cerebrovascular Segmentation, Local Binary Fitting, Hidden Markov Model, Maximum A Posterior

Posted Date: February 16th, 2021

DOI: https://doi.org/10.21203/rs.3.rs-198233/v1

License: (c) (i) This work is licensed under a Creative Commons Attribution 4.0 International License. Read Full License 


\title{
Cerebrovascular Segmentation Based on Hidden Markov Model in Phase-Contrast Angiography Images
}

\author{
Kangneng Zhou ${ }^{1}$, Cheng Chen ${ }^{1}$, Yuanyuan $\mathrm{Lu}^{2}$, Xinmeng Guo ${ }^{1}$, Wubin $\mathrm{Li}^{1}$, Huilin Wang ${ }^{1}$, \\ Xintong $\mathrm{Wu}^{1}$, Ruoxiu Xiao ${ }^{1,3, *}$
}
${ }^{1}$ School of Computer and Communication Engineering, University of Science and Technology Beijing, Beijing 100083, China
${ }^{2}$ Department of Ultasound, Chinese PLA General Hospital, Beijing, 100853, China
${ }^{3}$ Institute of Artificial Intelligence, University of Science and Technology Beijing, Beijing 100083, China

*Author correspondence: xiaoruoxiu@ ustb.edu.cn

\begin{abstract}
:
Background: Phase-Contrast Angiography (PCA) is an acceptable magnetic resonance imaging method for cerebrovascular diseases diagnosis. However, it is an important and great challenge to accurately extract cerebrovascular structures from PCA images because of the complex vascular structures and large amount of noise. To accomplish this task, this work proposes a cerebrovascular segmentation algorithm based on Local Binary Fitting (LBF) and Hidden Markov Model (HMM), which can accurately extraction features from PCA data.

Results: Dice Similarity Coefficient (DSC), False Positive Score (FPN), and False Negative Score (FTN) are defined as metrics to assess this algorithm. Results show this method obtain higher accuracy $(74.58 \%, 4.93 \%, 24.48 \%)$ than compared methods.

Conclusion: Based on quantitative results, it appears that the proposed method has a higher accuracy rate compared to other methods. Due to no human correction and has no training process, it performs well on small datasets. Thus, this algorithm can accord with clinical requirements.
\end{abstract}


Key Words: Cerebrovascular Segmentation; Local Binary Fitting; Hidden Markov Model; Maximum A Posterior

\section{Background}

Cerebrovascular disease is a major threat to human's health [1]. Currently, it is diagnosed mainly by imaging techniques, such as Digital Subtraction Angiography (DSA), CT Angiography (CTA), and Magnetic Resonance Imaging (MRA). Phase-Contrast Angiography (PCA), whose results are based on phase change of blood stream due to interaction between flowing blood and gradient field, is a novel MRA technique without need for contrast media injection [2]. Comparing to other MRA techniques, it has a larger imaging area and can present both arterial and venous structures. Usually, features of PCA images are very complicated due to small and complex vessels and susceptibility to hemodynamics. Therefore, accurate cerebrovascular segmentation from PCA images is still a difficult and important task.

Nowadays, researchers have presented many cerebrovascular segmentation algorithms [3]. There are three kinds of different algorithms according to their principles: segmentation algorithms based on gray-scale of vessels, shape of vessels, and deep learning.

Algorithms based on gray-scale of vessels are feasible because vessels are bright in images [4], which means they have appropriate contrast with rest part of images. However, how to deal with noise and other issues need to be solved urgently. Rui et al. [5] calculated foreground threshold based on statistical distribution of foreground and background pixels, which were obtained by threshold method, and extract vessels from images. Hassouna et al. [6] proposed a cerebrovascular segmentation algorithm based on a statistical model, which obtained segmentation results by fitting brain images with a Rayleigh distribution and two normal distributions with fitting parameters estimated by Expectation Maximization (EM [7]). Although these algorithms are simple and efficient, they are not only sensitive to noise and gray-scale diversity, but also unable to deal with complex situations where images have blurred borders and rupture of vessels because neglection of spatial characteristics.

Because tubular structure is an important spatial characteristic of vessels, some researchers model vessels and then separate them. For example, Carrilloz et al. [8] 
proposed an algorithm, which analyzes binary connected components on surface of a sphere moving along a vessel, and recursively tracks branches and detects branches, to obtain surface of vessels. Friman et al. [9] proposed a parameterized vascular segment tracking and extraction method, which achieves vascular segmentation by establishing a gray-scale model of vascular section and a parameterized model of vascular segment, and then detects and fits candidate vascular region in tracking direction.

With widespread application of deep learning in medical image processing, vessel segmentation can also be achieved by deep learning methods, especially Convolutional Neural Networks (CNN). For example, two models based on improved U-Net [10] and DeepVesselNet [11] networks respectively gave great performances in cerebrovascular segmentation. However, algorithms based on deep learning requires a large amount of data with consistent imaging parameters for training and testing. If dataset is too small, overfitting is likely to occur.

Therefore, this work proposes a cerebrovascular segmentation algorithm combining Local Binary Fitting (LBF) [12] and Hidden Markov Model (HMM) [13], which combines advantages of algorithms based on gray features and shape of vessels. The specific steps are as follows. First, images were denoised by anisotropic smooth preprocessing while preserving edges of vessels. Then, coarse segmentation results are obtained by LBF level set segmentation method. Finally, fine segmentation results are obtained by HMM based on the coarse-level results. In HMM, Gaussian distribution parameters are initialized first by K-means. Then, probabilities are calculated by EM algorithm and transformed into the optimization of the energy function. Finally, fine segmentation results are obtained by determining whether voxels belong to vessels or background using MAP algorithm.

\section{Results}

Ten clinical cases from open head magnetic resonance data set were randomly selected and tested in this study to evaluate the feasibility of the proposed method. Data were scanned by Siemens Biograph mMR equipment. The scanning parameters are $120 \mathrm{KV}$ voltage and $150 \mathrm{~mA}$ current. The actual size interval is $0.5 \mathrm{~mm} \times 0.5 \mathrm{~mm} \times 0.8 \mathrm{~mm}$, and the corresponding pixel size is $400 \times 400 \times 330$. Each clinical expert manually marks the cerebrovascular as ground truth for evaluation. 
Maximum Intensity Projection (MIP) [14] is used to visually evaluate segmentation results. The basic idea of MIP is to select a projection direction, then take the maximum gray scale as the projection value, and finally achieve the maximum brightness on the plane. The projection shows the three-dimensional information of vessels because the vessels are bright contrasted to the background.
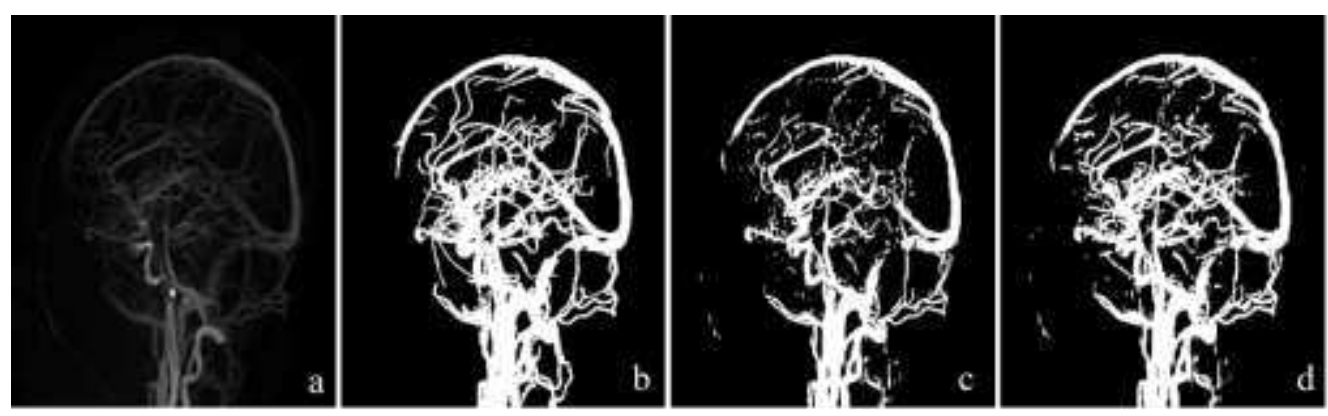

Figure 1. MIP projection of cerebrovascular segmentation results on a plane. (a). Original image. (b). Result of manual segmentation (ground truth). (c). Result of LBF segmentation. (d). Result of the proposed method.
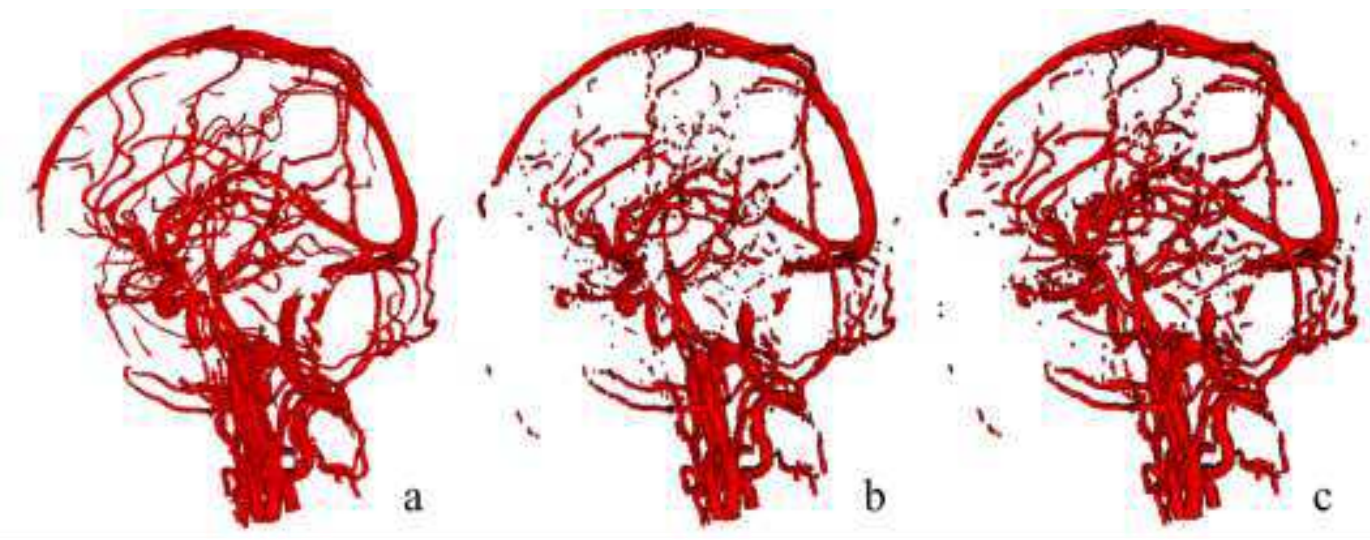

Figure 2. Three-dimensional Renderings of the segmented cerebrovascular. (a). Result of manual segmentation (ground truth). (b). Result of LBF segmentation. (c). Result of the proposed method.

Figure 1 shows the projections of cerebrovascular segmentation results on a plane. Figure 1(b) (ground truth) is continuous and smooth, and the vessels can completely match to those of the original data. 
LBF algorithm is mainly based on the information of local images, which is the weighted average of gray scale in the Gaussian window outside the contour when the local energy function converges. When LBF algorithm processes an image with uneven gray scale, the curve terminates early in convergence, which leads to over-segmentation of the result (Figure 1(c)). In addition, $\mathrm{LBF}$ algorithm lack the ability to judge whether a pixel of the edge vessel belongs to vascular or non-vascular area, which leads to misjudgment and vascular rupture (Figure 1(c)).

HMM algorithm obtains the initial parameters by clustering, and establishes the state set from the feature field to the label field. In this way, the energy function can get more vessel information. Figure 1(d) shows that the contour and the continuity of the vessels is similar to the ground truth. It can be seen that under this random field, the energy curve established based on the vessel information can finally converge to the expected value, which means that the HMM random field can fit correctly when processing cerebral vessel image.

As shown in Figure 2, 3D rendering is introduced to analysis those results. In Figure 2, (a), (b) and (c) represent the results of ground truth, LBF and the proposed method, respectively. It can be seen that the ground truth is very smooth, while the result of LBF has a lot of fractures. On the contrary, the result of the proposed method is similar to the ground truth. Therefore, the three-dimensional rendering shows the same result as twodimensional MIP projection. Figure 3 shows an enlarged view of 3D segmentation details. It can be clearly seen that LBF will ignore many boundaries in segmentation, especially the part of small vessels. The proposed method can fill these segmentation deficiencies, and it also has better performance in local small vessels. It is worth mentioning that segmentation of the proposed method may cause some over-segmentation. 

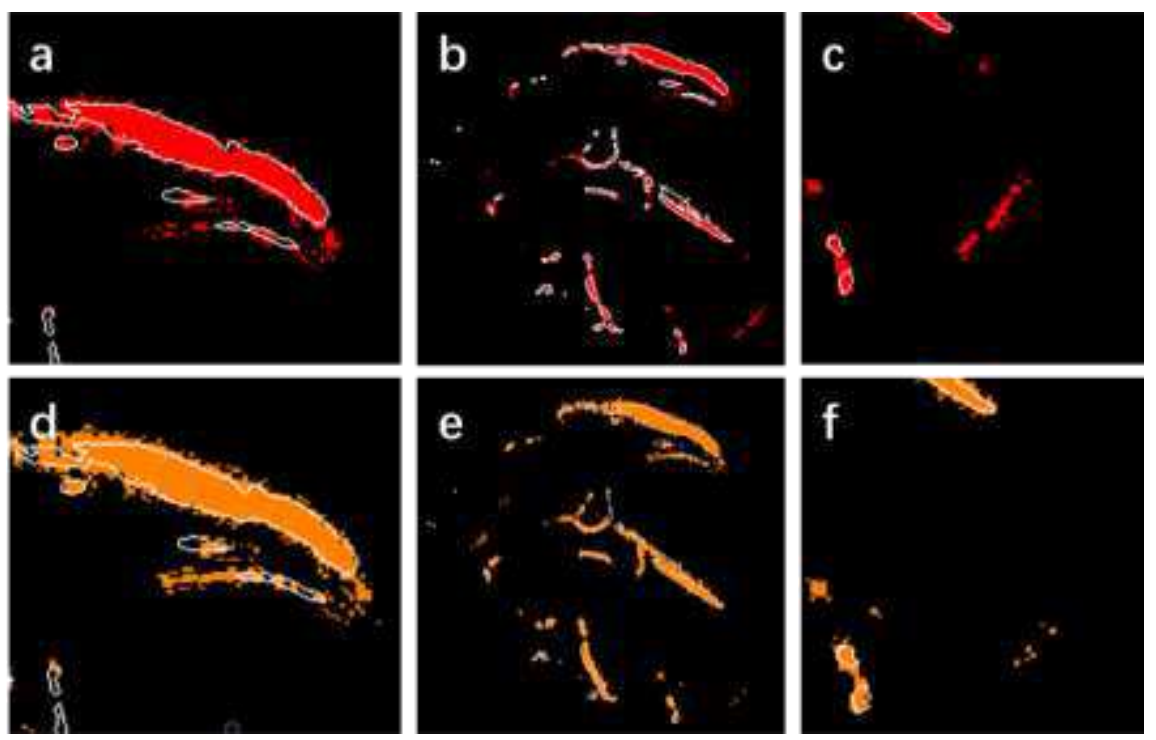

Figure 3. Segmentation details of LBF, and the proposed method. The red areas of a, b, and $\mathrm{c}$ are the LBF segmentation results, and the yellow areas of $\mathrm{d}, \mathrm{e}$, and $\mathrm{f}$ are the proposed method results. The white outline is ground truth.

To evaluate segmentation results quantitatively, the obtained results are evaluated by terms of Dice Similarity Coefficient (DSC), False Positive Score (FPN), and False Negative Score (FTN) [15]. DSC, FPN and FTN respectively quantify the accuracy, oversegmentation rate and under-segmentation rate. The evaluation method is as follows:

$$
\left\{\begin{array}{l}
D S C=2\left(R_{\text {Seg }} \cap R_{G T}\right) /\left(R_{\text {Seg }}+R_{G T}\right) \\
F P N=O_{S} /\left(R_{\text {Seg }}+R_{G T}\right) \\
F T N=U_{S} /\left(R_{\text {Seg }}+R_{G T}\right)
\end{array}\right.
$$

In (1), $R_{\text {Seg }}$ represents the segmentation result, and $R_{G T}$ represents ground truth. $O_{S}$ represents the part of the segmentation result that exceeds the ground truth, while $U_{S}$ represents the part of the ground truth that exceeds the segmentation result.

Evaluation results are shown in Table 1. The results show that DSC, FPN, FTN obtained by LBF and the proposed method are $69.09 \%, 23.97 \%, 14.57 \%$ and $74.58 \%$, $4.92 \%, 24.48 \%$, respectively. Ours method is $5.49 \%$ higher on DSC than LBF, and the segmentation is more accurate. At the same time, the FPN of LBF reaches $23.97 \%$ because of insufficient information characteristics, which leads to the early termination of the curve 
in curve approximation, and eventually leads to thicker vessels in segmentation results than the actual situation. For a more intuitive display, Figure 4 plots DSC results of the two segmentation methods. In this statistical histogram, D3 and D5 of this method show significantly higher performance than LBF. D5 with the largest difference is nearly $24.01 \%$ higher. But D4, D6 and D7 are close in the DSC. This is because their FTN of ours is higher than others, showing some redundant segmentation boundaries, leading to incorrect segmentation. Although the FTN of our approach is larger than LBF, it is in the clinically acceptable range. Therefore, reasonable parameter adjustments are needed in actual application.

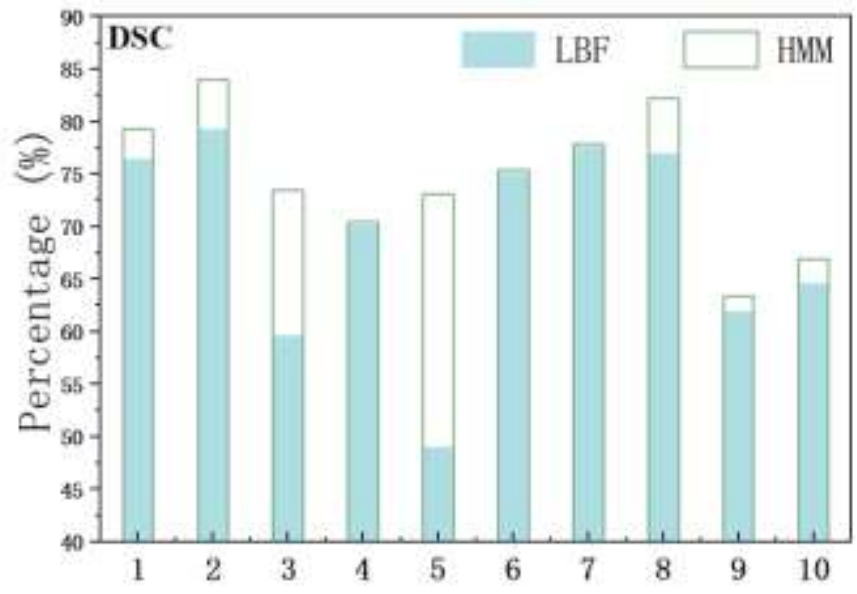

Figure 4. Statistical histogram of DSC. Where, rectangle with a green frame is DSC value of the proposed method. Its blue fills are DSC value of LBF.

In addition, in order to verify the method in this work, this work designed a 3D-U-Net [16] model as a comparative experiment. A cross-validation method is applied: D1-D5 is selected as the training, and D6-D10 is used as the testing for comparison. Then training and testing set are exchanged, and the comparison is performed again. Table 2 shows the quantitative results. In terms of performance, the proposed method has achieved better performance in both sets of tests. From the data distribution, although the results of 3D-UNet obtain $0.05 \%$ PFN, the FTN is over $48 \%$. This is because the insufficient amount of data leads to the insufficient extraction of vascular features by the neural network, which will lead to serious clinical misdiagnosis results, and there is no way to avoid the risk of 
surgery. Therefore, this work has the advantages of not relying on large amounts of data, fully automatic, and high performance. It has certain clinical potential.

Table1. The Analysis of LBF and the proposed method

\begin{tabular}{ccccccc}
\hline & \multicolumn{3}{c}{ LBF } & & \multicolumn{2}{c}{ Ours } \\
& DSC & FPN & FTN & DSC & FPN & FTN \\
\hline D1 & 0.7643 & 0.2333 & 0.1620 & 0.7926 & 0.0497 & 0.2362 \\
D2 & 0.7928 & 0.2431 & 0.1169 & 0.8395 & 0.0651 & 0.1845 \\
D3 & 0.5968 & 0.1966 & 0.2065 & 0.7343 & 0.0547 & 0.2110 \\
D4 & 0.7019 & 0.2007 & 0.0975 & 0.7040 & 0.0115 & 0.2845 \\
D5 & 0.4906 & 0.4359 & 0.0735 & 0.7307 & 0.0868 & 0.1825 \\
D6 & 0.7514 & 0.1961 & 0.2009 & 0.7540 & 0.0371 & 0.2709 \\
D7 & 0.7763 & 0.1688 & 0.1915 & 0.7784 & 0.0473 & 0.2488 \\
D8 & 0.7695 & 0.2746 & 0.1212 & 0.8224 & 0.0764 & 0.1961 \\
D9 & 0.6196 & 0.2435 & 0.1369 & 0.6333 & 0.0457 & 0.3210 \\
D10 & 0.6461 & 0.2043 & 0.1496 & 0.6690 & 0.0184 & 0.3127 \\
AVG & 0.6909 & 0.2397 & $\mathbf{0 . 1 4 5 7}$ & $\mathbf{0 . 7 4 5 8}$ & $\mathbf{0 . 0 4 9 3}$ & 0.2448 \\
\hline
\end{tabular}

Table 2. The Analysis of 3D-Unet (D1-D10)

\begin{tabular}{cccc}
\hline & \multicolumn{3}{c}{ 3D-Unet } \\
\cline { 2 - 4 } & DSC & FPN & FTN \\
\hline D1 & 0.7451 & 0.0008 & 0.3350 \\
D2 & 0.6944 & 0.0008 & 0.3933 \\
D3 & 0.7100 & 0.0009 & 0.3669 \\
D4 & 0.7536 & 0.0010 & 0.2700 \\
D5 & 0.3242 & 0.0001 & 0.8055 \\
D6 & 0.6554 & 0.0002 & 0.4856 \\
D7 & 0.5236 & 0.0003 & 0.6416 \\
D8 & 0.6742 & 0.0003 & 0.4518 \\
D9 & 0.6344 & 0.0009 & 0.5016 \\
D10 & 0.4792 & $\mathbf{0 . 0 0 0 5}$ & 0.6231 \\
AVG & 0.6194 & 0.0493 & 0.4874 \\
Ours & $\mathbf{0 . 7 4 5 8}$ & & $\mathbf{0 . 2 4 4 8}$ \\
\hline
\end{tabular}




\section{Conclusions}

This work proposes a cerebrovascular segmentation algorithm based on HMM. It extracts the vessels' contour features from the energy function established in LBF combining with the weighted average of local gray scale. Then, the state sets of the characteristic field and the labeled field is obtained by establishing a hidden Markov

random field, and the energy function equation is generalized. The hidden Markov random field is initialized by K-means, and updated by the EM algorithm until the a priori energy function converges. The segmentation results are then obtained.

Ten sets of data tested this method. The results show that the vascular structure segmented by proposed method is close to the manual segmentation. In terms of visualization, the results of the LBF mismatched certain brain tissue into vessels, and the segmented vessel results were thinner than the real results. After processing by the proposed method, the edge of the segmented vessel was getting closer to the real vessel, and some mismatch points were eliminated. In terms of quantitative results, the proposed method achieves higher accuracy than LBF. Therefore, proposed method does not require any manual intervention and can meet actual needs. It is worth noting that there is currently no published paper that applies the proposed method in medical image processing to build models. This paper makes the first exploration, puts forward corresponding methods and test them by experiments, which provides a new direction for the analysis of cerebrovascular images.

\section{Methods}

This section is organized to introduce the methods proposed in this work. Diagram of proposed cerebrovascular segmentation algorithm is shown in Figure 5. In this work, original images were denoised by anisotropic filter. Then, level set segmentation is performed using LBF, Finally, segmentation results are obtained by our proposed method based on HMM. 


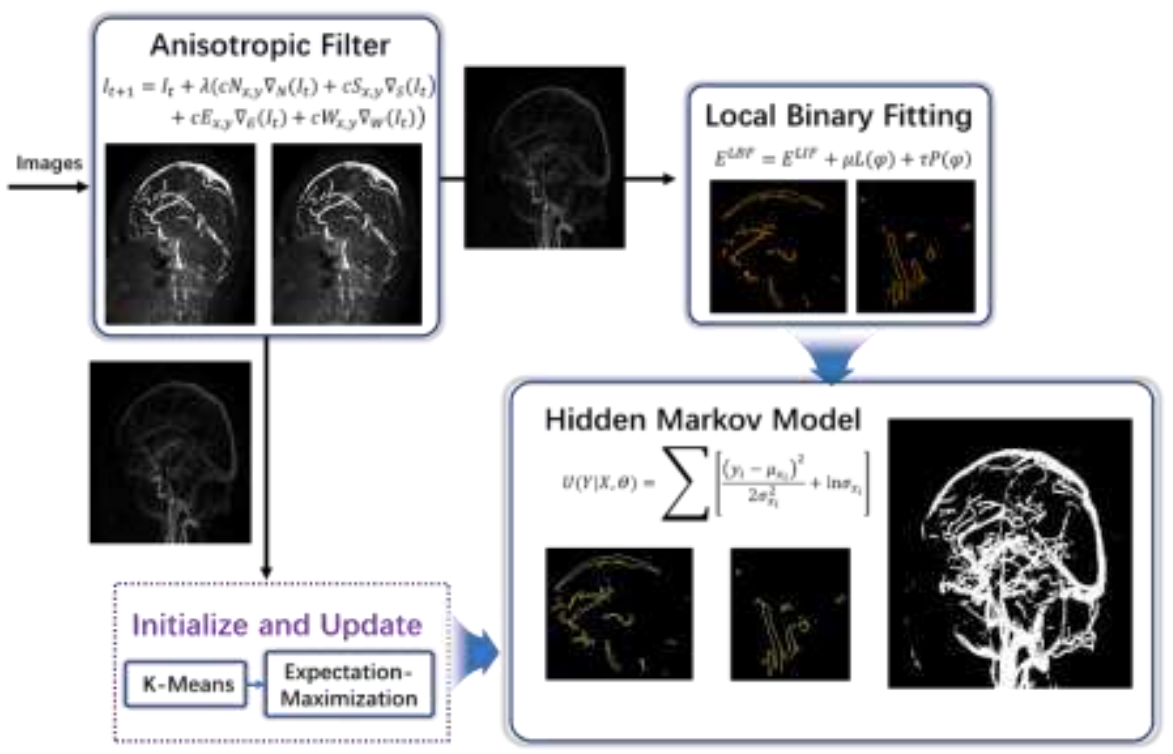

Figure 5. Diagram of the proposed method

Anisotropic Filter

Noise and interference in medical images [17] are evitable, especially in PCA images. There are a large number of tubular structures in images because of flow, which has a bad affect in cerebrovascular segmentation. Mean filter, median filter, and Gaussian filter are three common filters. Mean filter can only weaken noise instead of eliminating it. Median filter can cause discontinuity of vessel because of its strong denoising ability. Gaussian filter blurs edges of vessels, which leads to a decrease in accuracy of segmentation.

Anisotropic filter is based on partial differential equations [18]. It was first proposed based on anisotropic diffusion theory of heat. Then it was introduced to image processing and applied to noise suppression and edge detection. The basic idea of anisotropic filtering is to perform large-scale smoothing in flat area of the image while perform small-scale smoothing in edge area. In this way, edges in the image are protected while the noises are suppressed. Let $I$ be an image, its diffusion model is as follows:

$$
\left\{\begin{array}{c}
\frac{\partial \mathrm{I}}{\partial \mathrm{t}}=\operatorname{div}[c(\|\nabla I\|) \cdot \nabla I] \\
I(t=0)=I_{0}
\end{array}\right.
$$


$\operatorname{div}(\cdot)$ represents the divergence operator while $\nabla(\cdot)$ represents the gradient operator. $\|\nabla I\|$ is the amplitude of the gradient of the image, $c(\|\nabla I\|)$ is diffusion function, and $\mathrm{t}$ is number of iterations.

Diffusion at pixel $\mathrm{p}$ can be expressed as:

$$
\mathrm{I}_{p}^{t+1}=\mathrm{I}_{p}^{t}+\frac{\lambda}{\left|\eta_{p}\right|} \sum_{q \in \eta_{p}} c\left(\nabla I_{p, q}^{t}\right) \nabla I_{p, q}^{t}
$$

$\mathbf{I}_{p}^{t}$ is the gray scale at pixel $\mathrm{p}$ after $\mathrm{t}$ times of iteration. $\lambda$ is a constant controlling the overall intensity of diffusion, and $\eta_{p}$ is the size of the neighborhood space. It can be seen from Eq. (3) that anisotropic filter is a kind of iteration. Figure 6 shows the denoising results of a PCA image when $\lambda$ is $0.2,0.4,0.6,0.8,1,1.5,2$ respectively. As shown in Figure 6 , the larger $\lambda$ is, the stronger the denoising ability is. However, when $\lambda$ increases to 1.5 and 2 , the vessel is destroyed. Therefore, the diffusion coefficient $\lambda$ of this work is 1.
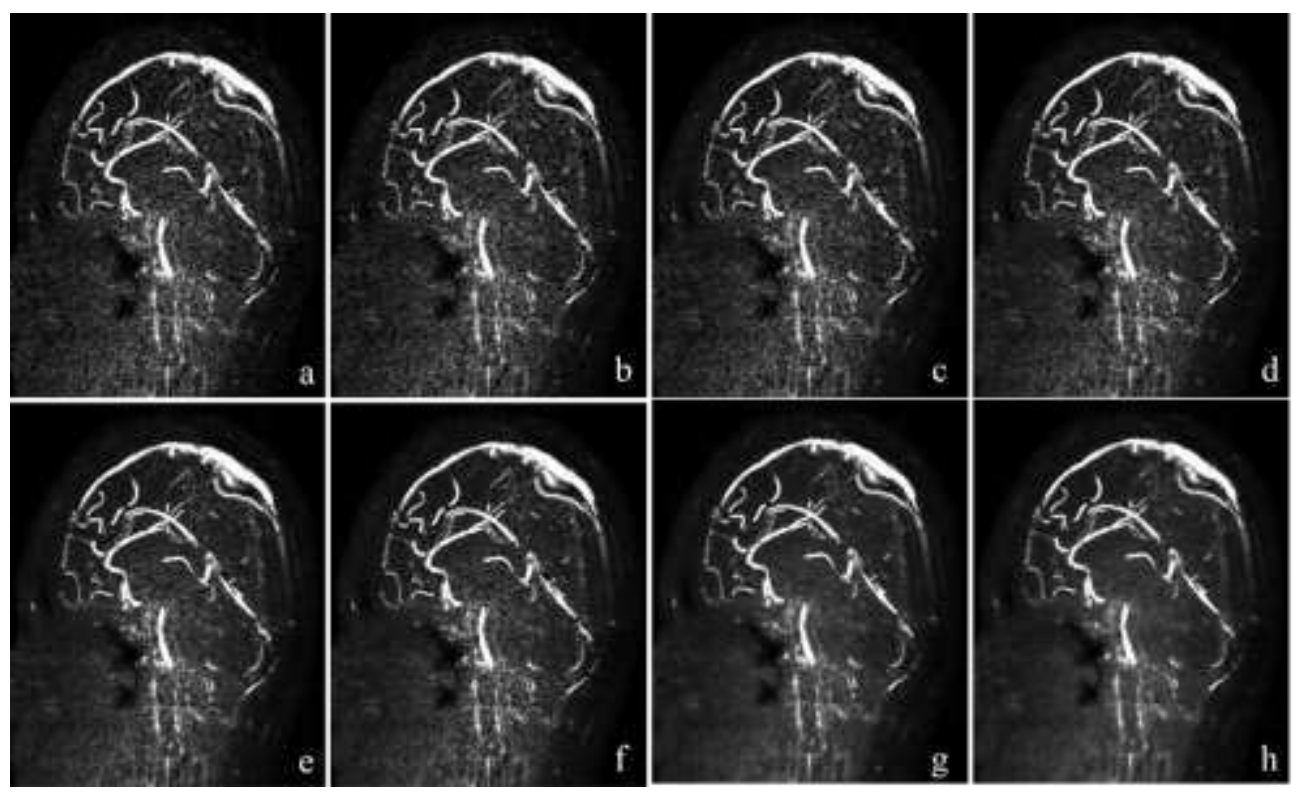

Figure 6. Results of anisotropic filter. a. The original image. $\mathrm{b}-\mathrm{h}$. The denoising results when denoising coefficient $\lambda$ is $0.2,0.4,0.6,0.8,1,1.5$, and 2 respectively. 
Coarse Segmentation Based on Local Binary Fitting

Active contour models have been widely used in image segmentation. The current active contour segmentation models can be divided into two categories: edge-based models [19] and region-based models. Region-based segmentation models [20] determines each target region through a certain region descriptor, thereby directing the active contour to move until it converges to the segmentation boundary. It can receive great results when the input images have strong pixel consistency in the target area.

LBF algorithm in this work is a region-based active contour model algorithm [21], which constructs a region-based active contour model using a variational level set equation. A fitted scalable region energy function is defined by the contour and two image brightness fitting functions around the contour. The best results can be obtained when the fitting function represents the average of the brightness around the contour. A kernel function with a scale parameter ensures the scalability. The brightness of the region within a scale can be used to extend from the surrounding pixels to the whole image. This energy is then assigned to a variational level set equation with the level set normalized. The minimization of the associated energy function is achieved by curve evolution. Two functions are calculated and fitted with brightness at a certain scale, and the contour movement is induced to the boundary of the image.

The functional form of the local fitting energy represented by the LBF level set is:

$$
\begin{aligned}
& \varepsilon^{F i t}\left(\varphi, f_{1}(x), f_{2}(x)\right)=\sum_{i=1}^{2} \lambda_{i} \int_{\Omega} K_{\delta}\left|I-f_{i}(x)\right|^{2} M_{i}(\phi) d x d y \\
& \text { In Eq. (4), } M_{1}(\varphi)=H(\varphi), M_{2}(\varphi)=1-H(\varphi)
\end{aligned}
$$

$f(x)$ is the local fit of the image at point $\mathrm{x}$, and $K_{\delta}$ is the Gaussian kernel function when $\delta>0$.

To extend $x$ from current position to the whole image and guarantee the stability of the evolution of level set $\varphi$, a distance regularization term is added to the function. Therefore, the total energy function is: 


$$
\begin{aligned}
& E^{L B F}=\int_{\Omega} \varepsilon^{F i t} d x d y+\mu \int_{\Omega} \delta_{\varepsilon}(\varphi)|\nabla \varphi| d x d y+\tau \int_{\Omega} \frac{1}{2}(|\nabla \varphi|-1)^{2} d x d y \\
& =E^{L I F}+\mu L(\varphi)+\tau P(\varphi)
\end{aligned}
$$

$E^{L I F}$ represents local energy, and $L(\varphi)$ represents the length of curve. $P(\varphi)$ is the distance regularization term, which is also known as punishment term. $\mu$ and $\tau$ are two non-negative constants. $f_{1}$ and $f_{2}$ approximately equal to the weighted average of gray scale in Gaussian window outside the contour in LBF model. LBF model obtains a better result on image segmentation with uneven gray scale because it is based on part of the image instead of the whole image.

Figure 7(a) shows the cerebrovascular segmentation of Figure 6(f) using LBF. It shows that the coarse cerebrovascular segmentation can be obtained by LBF, which is the initial conditions for accurate segmentation in this paper.

Fine Segmentation Based on Hidden Markov Model

The work is based on such assumption that the grayscale distribution of the vessel and the background obeys the Gaussian distribution. Based on Bayesian theory, the Maximum A Posterior (MAP) algorithm is introduced to separate the segmentation results into a feature field and a labeling field to achieve cerebrovascular segmentation. By calculating a posterior probability, the objective function is determined. Therefore, the task of accurate cerebrovascular segmentation can be accomplished by finding the MAP. According to [22], the Markov and Gibbs distributions are equivalent. Therefore, the Markov random field can be replaced by the Gibbs random field. In this way, the calculation of MAP can be converted to the optimization of the minimum energy, which can cut down the amount of calculation. The traditional Markov random field is sensitive to pixel outliers, which causes inaccurate segmentation or uneven edges. Therefore, hidden Markov random field is used instead of the traditional one.

Although the vessels are small, there must be hidden parameters. The observed parameter sequence and the hidden parameter sequence are probability-related. Therefore, a hidden Markov process and an observable set of states associated with this hidden Markov process are modeled. 
Dataset are defined as:

$$
\mathrm{Y}=\left(y_{1 * 1}, \ldots, y_{m * n}\right)
$$

$m, n$ are the numbers of rows and columns respectively. $y_{i * j}$ represents the gray scale of pixel in the i-th row and j-th column. Segmentation results are:

$$
X=\left(x_{1 * 1}, \ldots, x_{m * n}\right)
$$

In Eq. (7), $x \in L$. Images only consist of vessels and background, so this is a binary classification problem, which means $L=\{0,1\}$.

For MAP:

$$
X_{\mathrm{MAP}}=\arg \max \{P(Y \mid X) P(X)\}
$$

By adding the parameter of the Gaussian distribution, which is previously assumed, Eq. (8) is modified as:

$$
\mathbf{A}_{M A P}=\arg \max \{P(Y \mid X, \Theta) P(X)\}
$$

In Eq. (9), $P(X)$ can be replaced by the energy of Gibbs random field. $P(Y \mid X, \Theta)$ can be expanded as:

$$
P(Y \mid X, \Theta)=\prod P\left(y_{i} \mid x_{i}, \theta_{x_{i}}\right)
$$

In Eq. (10), $P\left(y_{i} \mid x_{i}, \theta_{x_{i}}\right)$ is a Gaussian distribution with parameter $\theta_{x_{i}}=\left(\mu_{x_{i}}, \sigma_{x_{i}}\right)$. In Markov random field, this parameter needs to be obtained through training. However, there is neither training nor a priori estimation in the hidden Markov random field. The EM algorithm is introduced to solve this. K-means, MAP, and EM algorithms are introduced to build a hidden Markov model [22] for fine segmentation of cerebrovascular.

K-means fuzzy clustering algorithm [23] is introduced to initialize the parameter $\Theta$, so an initial probability can be obtained at the beginning of the cerebrovascular segmentation algorithm. The data processed in this step is the coarse segmentation results after LBF, and the goal is to cluster the vessels to distinguish them from the background. K-means clustering algorithm is a classic distance-based clustering algorithm using distance as a criterion for similarity evaluation.

First, k pixels (vascular or non-vascular pixels) in the brain slice image are randomly selected as the clustering center. Then, each remaining pixel is normalized to the clustering 
set (cluster) closest to it based on the distance to the clustering center, and the average of each cluster is calculated to update the clustering center.

$$
E=\sum_{i=1}^{\mathrm{k}} \sum_{x \in C_{i}}\left|x-\bar{x}_{i}\right|^{2}
$$

In Eq. (11), $\mathrm{k}$ is the number of clusters, and $C_{i}$ is the i-th cluster. The output of the criterion function converges to a fixed value through iterations and it is parameter $\Theta$.

Each pixel has a position and gray scale in PCA images. K-means algorithm is not based on position, but on gray scale, which means pixels with similar gray scale are in a same cluster. Therefore, the parameter $\Theta$ in the EM algorithm can be initialized based on the previous LBF coarse segmentation results.

Then the images are processed by the EM algorithm. Traditional EM algorithm includes two major steps: E step (expectation) and $\mathrm{M}$ step (maximization). When E step works, set this iteration as the $\mathrm{t}$-th iteration. The vascular Gaussian distribution parameter set $\Theta_{t}$ is calculated in the previous step. This step is to calculate the likelihood function $Q\left(\Theta \mid \Theta_{t}\right)$ of the vascular segmentation.

$$
Q\left(\Theta \mid \Theta_{t}\right)=E\left(\log (P(\mathrm{X}, Y \mid \Theta)) \mid Y, \Theta_{t}\right)
$$

In M step, $Q\left(\Theta \mid \Theta_{t}\right)$ needs to be maximized as follows:

$$
\Theta_{t+1}=\arg \max \left(Q\left(\Theta \mid \Theta_{t}\right)\right)
$$

In the proceeding of segmentation, Eq. (12) and Eq. (13) are repeated until $\left\|\Theta_{t+1}-\Theta_{t}\right\|$ converges, which means the segmentation is stable. The parameter set $\Theta$ is obtained at this time. It is assumed that cerebrovascular obey the Gaussian distribution, so each parameter is defined with a Gaussian function. For parameter $\theta_{i}=\left(\mu_{i}, \sigma_{i}\right)$, the Gaussian function is defined as:

$$
G\left(z ; \theta_{i}\right)=\frac{1}{\sqrt{2 \pi \sigma_{i}^{2}}} \exp \left(-\frac{\left(z-\mu_{i}\right)^{2}}{2 \sigma_{i}^{2}}\right)
$$

A priori probability is defined as:

$$
P(X)=\frac{1}{Z} \exp (-U(X))
$$


In Eq. (15), $U(X)$ is a priori energy function.

$$
\begin{aligned}
& P(Y \mid X, \Theta)=\prod P\left(y_{i} \mid x_{i}, \theta_{x_{i}}\right) \\
& =\prod G\left(y_{i} ; \theta_{x_{i}}\right) \\
& =\frac{1}{Z^{\prime}} \exp (-U(Y \mid X))
\end{aligned}
$$

After initializing parameter $\Theta$ with K-means and calculating the probability distribution $P(y \mid x, \theta)$ by the EM algorithm, the label is predicted by MAP algorithm. The label is whether the pixel belongs to the cerebrovascular or background.

Bayes function is introduced:

$$
\boldsymbol{A}_{M A P}=\arg \max P(X \mid Y)=\arg \max P(Y \mid X) P(X)
$$

Take the logarithm of Eq. (17):

$$
\mathbf{A}_{M A P} \propto \arg \max (\ln P(Y \mid X)+\ln P(X))
$$

Bring Eq. (18) into the EM algorithm:

$$
\mathbf{A}_{M A P}=\arg \min (U(Y \mid X, \Theta)+U(X))
$$

The radicals of a second-order domain system is introduced to define $U(X)$ with potential energy:

$$
\begin{aligned}
& V_{c}\left(x_{i}, x_{j}\right)=\frac{1}{2}\left(1-I_{x_{i}, x_{j}}\right) \\
& I_{x_{i}, x_{j}}= \begin{cases}0 & \text { if } x_{i} \neq x_{j} \\
1 & \text { if } x_{i} \neq x_{j}\end{cases} \\
& U(x)=\sum V_{c}(x)
\end{aligned}
$$

For $U(Y \mid X)$, it is assumed that all pixels in vessels in the cerebrovascular image are independent of each other and obey Gaussian distribution.

$$
\begin{aligned}
& U(Y \mid X, \Theta)=\sum U\left(y_{i} \mid x_{i}, \Theta_{x_{i}}\right) \\
& =\sum\left[\frac{\left(y_{i}-\mu_{x_{i}}\right)^{2}}{2 \sigma_{x_{i}}^{2}}+\ln \sigma_{x_{i}}\right]
\end{aligned}
$$

In Eq. (21), a fixed output can be obtained through iterations. 
As is assumed in the previous section, the cerebrovascular distribution is based on a Gaussian distribution, and the parameter is based on a single Gaussian distribution parameter $\theta_{x_{i}}=\left(\mu_{x_{i}}, \sigma_{x_{i}}\right)$. It means that the intensity distribution of the cerabrovascular obeys a single Gaussian distribution. However, the intensity distribution of actual cerebrovascular is complicated, so it is imprecise to assume that the cerebrovascular distribution is based on a Gaussian distribution. In medical image processing, many methods have been proposed to improve the segmentation of cerebrovascular images. The Gaussian mixture model is a typical one, which is simple and effective. This method applies a statistical model to medical images according to the pixel features. Therefore, a Gaussian Mixture Model (GMM) [24] is proposed to fit the intensity distribution of cerebrovascular. Comparing with the previous model, GMM is more complex and powerful. It can match to the actual situation better.

The parameters of a GMM with i members are:

$$
\theta=\left\{\left(\mu_{1}, \sigma_{1}\right),\left(\mu_{2}, \sigma_{2}\right), \ldots,\left(\mu_{i}, \sigma_{i}\right)\right\}
$$

The Gaussian function now is the weighted sum of the Gaussian distribution function corresponding to each parameter.

$$
G(z ; \theta)=\sum w_{j} G\left(z ; \theta_{j}\right)
$$

GMM revises the EM algorithm. The parameter of the Gaussian distribution at this time is determined by the calculation of E-step, and the parameters of the Gaussian mixture model is calculated by M-step.

Figure 7(b) is the result of the proposed method. As shown in Figure 7(b), after the HMM is improved, the segmentation result is more accurate than the result of LBF, and it shows great continuity of vessels. 

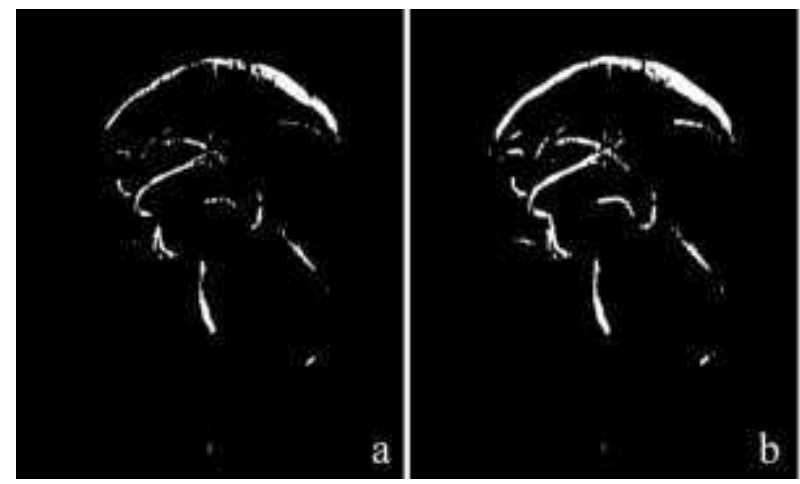

Figure 7. Cerebrovascular segmentation results of Figure6f. (a). Result of LBF segmentation. (b). Result of the proposed method.

\section{Ethics approval and consent to participate}

Not applicable.

\section{Consent for publication}

Not applicable.

\section{Competing interests}

The authors declare that they have no competing interest.

\section{Funding}

This work was supported by the National Science Foundation Program of China (61701022), the National Key Research and Development Program of China Under Grant (2016YFC0901304), Beijing Natural Science Foundation (L202030), Major Science and Technology Project of Zhejiang Province Health Commission(WKJ-ZJ-2112), the Fundamental Research Funds for the Central Universities(FRF-DF-20-05), and the Beijing Top Discipline for Artificial Intelligent Science and Engineering, University of Science and Technology Beijing.

\section{Authors' contributions}

Kangneng Zhou is responsible for methodology, software, validation and writing-original draft. Cheng Chen is responsible for conceptualization, visualization and writingreviwe\&editing. Yuanyuan Lu is responsible for data curation and resources. Xinmeng Guo is responsible for writing-reviwe\&editing and investigation. Wubin $\mathrm{Li}$ is responsible for methodology. Huilin Wang is responsible for writing-reviwe\&editing. Xintong Wu is responsible for writing-reviwe\&editing. Ruoxiu Xiao is responsible for funding acquisition, project administration, resources, supervison and writing-reviwe\&editing. 


\section{Acknowledgement}

The authors thank all subjects who participated in this study.

\section{Availability of data and material}

All material cited in this article has been taken from publicly available sources. The data used in this article is private.

\section{References}

[1]. Tonomura Shuichi, Ihara Masafumi, Friedland Robert P. Microbiota in cerebrovascular disease: A key player and future therapeutic target. Journal of cerebral blood flow and metabolism : official journal of the International Society of Cerebral Blood Flow and Metabolism, 2020, 40(7): 1368-1380.

[2]. Wang Yuzhao, Gao Duo, Liu Huaijun. Evaluation of Cerebral Blood Flow Dynamics in Transient Ischemic Attacks Patients with Fast Cine Phase Contrast Magnetic Resonance Angiography. Computational and mathematical methods in medicine, 2020, 2020(4097829):6.

[3]. Lv Zhilong, Mi Fubo, Wu Zhongke, et al. A Parallel Cerebrovascular Segmentation Algorithm Based on Focused Multi-Gaussians Model and Heterogeneous Markov Random Field. IEEE transactions on nanobioscience, 2020, 19(3): 538-546.

[4]. Neira M , A. Prüss-Ustün. Preventing disease through healthy environments: A global assessment of the environmental burden of disease. Toxicology Letters, 2016, 259:S1.

[5]. Wang R, Li C, Wang J, et al. Threshold segmentation algorithm for automatic extraction of cerebrovascular from brain magnetic resonance angiography images. Journal of neuroscience methods, 2015, 241: 30-36.

[6]. Hassouna M S, Farag A A, Hushek S, et al. Cerebrovascular segmentation from TOF using stochastic models. Medical image analysis, 2006, 10(1): 2-18.

[7]. Lili Yu, Ding-Geng Chen, Jun Liu. Efficient and direct estimation of the variancecovariance matrix in EM algorithm with interpolation method. Journal of Statistical Planning and Inference, 2021, 211: 119-130. 
[8]. Carrillo J F, Hoyos M H, Dávila E E, et al. Recursive tracking of vascular tree axes in 3D medical images. International Journal of Computer Assisted Radiology and Surgery, 2007, 1(6): 331-339.

[9]. Friman O , Hindennach M , Caroline Kühnel, et al. Multiple hypothesis template tracking of small 3D vessel structures. Medical Image Analysis, 2010, 14(2):160-171.

[10]. Livne M, Rieger J, Aydin O U, et al. A U-Net Deep Learning Framework for High Performance Vessel Segmentation in Patients With Cerebrovascular Disease. Frontiers in neuroscience, 2019, 13:97.

[11]. Tetteh G, Efremov V, Forkert N D, et al. Deepvesselnet: Vessel segmentation, centerline prediction, and bifurcation detection in 3-d angiographic volumes. arXiv preprint arXiv:1803.09340, 2018.

[12]. Li C, Kao C Y, Gore J C, et al. Implicit active contours driven by local binary fitting energy:2007 IEEE Conference on Computer Vision and Pattern Recognition. IEEE, 2007: $1-7$.

[13]. Yuan Yao,Yi Cao. A Neural network enhanced hidden Markov model for tourism demand forecasting. Applied Soft Computing Journal,2020,94(106465).

[14]. Xiao R, Ding H, Zhai F, et al. Cerebrovascular segmentation of TOF-MRA based on seed point detection and multiple-feature fusion. Computerized Medical Imaging and Graphics, 2018, 69: 1-8.

[15]. Cheng Chen, Ruoxiu, Xiao, Tao Zhang, et al. Pathological lung segmentation in chest CT images based on improved random walker. Computer Methods and Programs in Biomedicine, 2020, 105864.

[16]. Özgün Çiçek, Ahmed Abdulkadir, Soeren S. Lienkamp, et al. 3D U-Net: Learning Dense Volumetric Segmentation from Sparse Annotation. Medical Image Computing and Computer-Assisted Intervention - MICCAI 2016, 2016, 424-432. 
[17].Asim ur Rehman Khan, Farrokh Janabi-Sharifi, Mohammad Ghahramani, et al. Comparison of Reducing the Speckle Noise in Ultrasound Medical Images using Discrete Wavelet Transform. 2019, 10(5):340-350.

[18].Tang Chen, Wang Linlin, Yan Haiqing. Overview of anisotropic filtering methods based on partial differential equations for electronic speckle pattern interferometry. Applied optics, 2012, 51(20): 4916-4926.

[19].Xiaofeng Luo, Junyuan Yang, Zhen Jin, et al. An edge-based model for nonMarkovian sexually transmitted infections in coupled network. 2020, 13(02):35.

[20].Appia Vikram, Yezzi Anthony. Active Geodesics: Region-based Active Contour Segmentation with a Global Edge-based Constraint. Proceedings. IEEE International Conference on Computer Vision, 2011, 2011: 1975-1980.

[21].Besag J. Spatial Interaction and the Statistical Analysis of Lattice Systems. Journal of the Royal Statistical Society: Series B (Methodological), 1974, 36(2).

[22].Wang Q. GMM-based hidden Markov random field for color image and 3D volume segmentation. arXiv preprint arXiv:1212.4527, 2012.

[23].Wiharto Wiharto, Suryani Esti. The Comparison of Clustering Algorithms K-Means and Fuzzy C-Means for Segmentation Retinal Blood Vessels. Acta informatica medica : AIM : journal of the Society for Medical Informatics of Bosnia \&amp; Herzegovina : casopis Drustva za medicinsku informatiku BiH, 2020, 28(1): 42-47.

[24].Singhal Amit, Singh Pushpendra, Lall Brejesh, et al. Modeling and prediction of COVID-19 pandemic using Gaussian mixture model. Chaos, solitons, and fractals, 2020, 138(110023). 


\section{Figures}
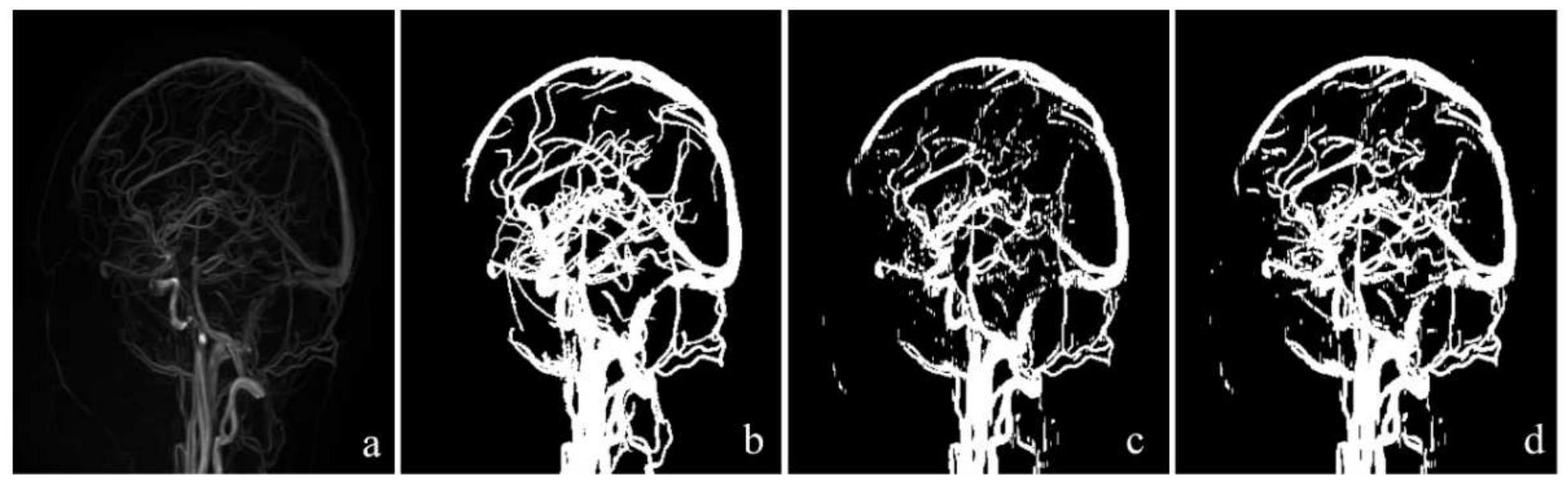

Figure 1

MIP projection of cerebrovascular segmentation results on a plane. (a). Original image. (b). Result of manual segmentation (ground truth). (c). Result of LBF segmentation. (d). Result of the proposed method.
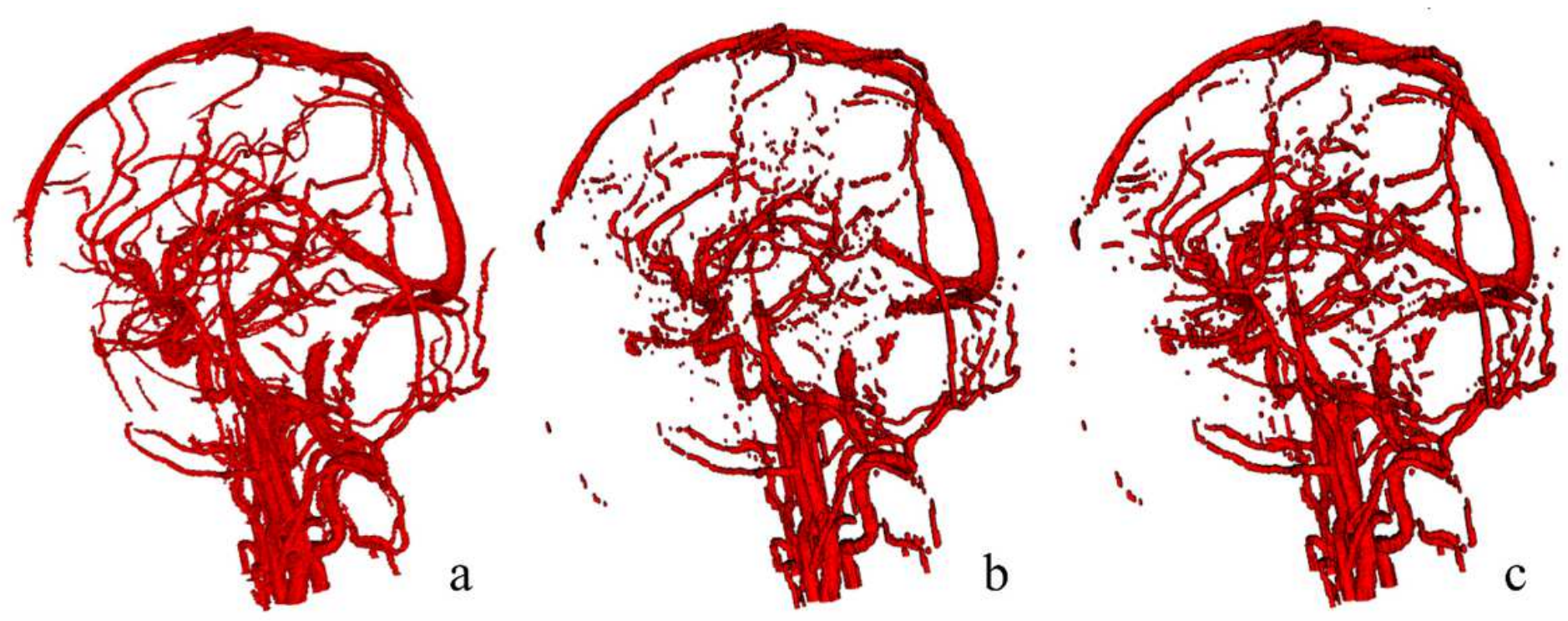

Figure 2

Three-dimensional Renderings of the segmented cerebrovascular. (a). Result of manual segmentation (ground truth). (b). Result of LBF segmentation. (c). Result of the proposed method. 

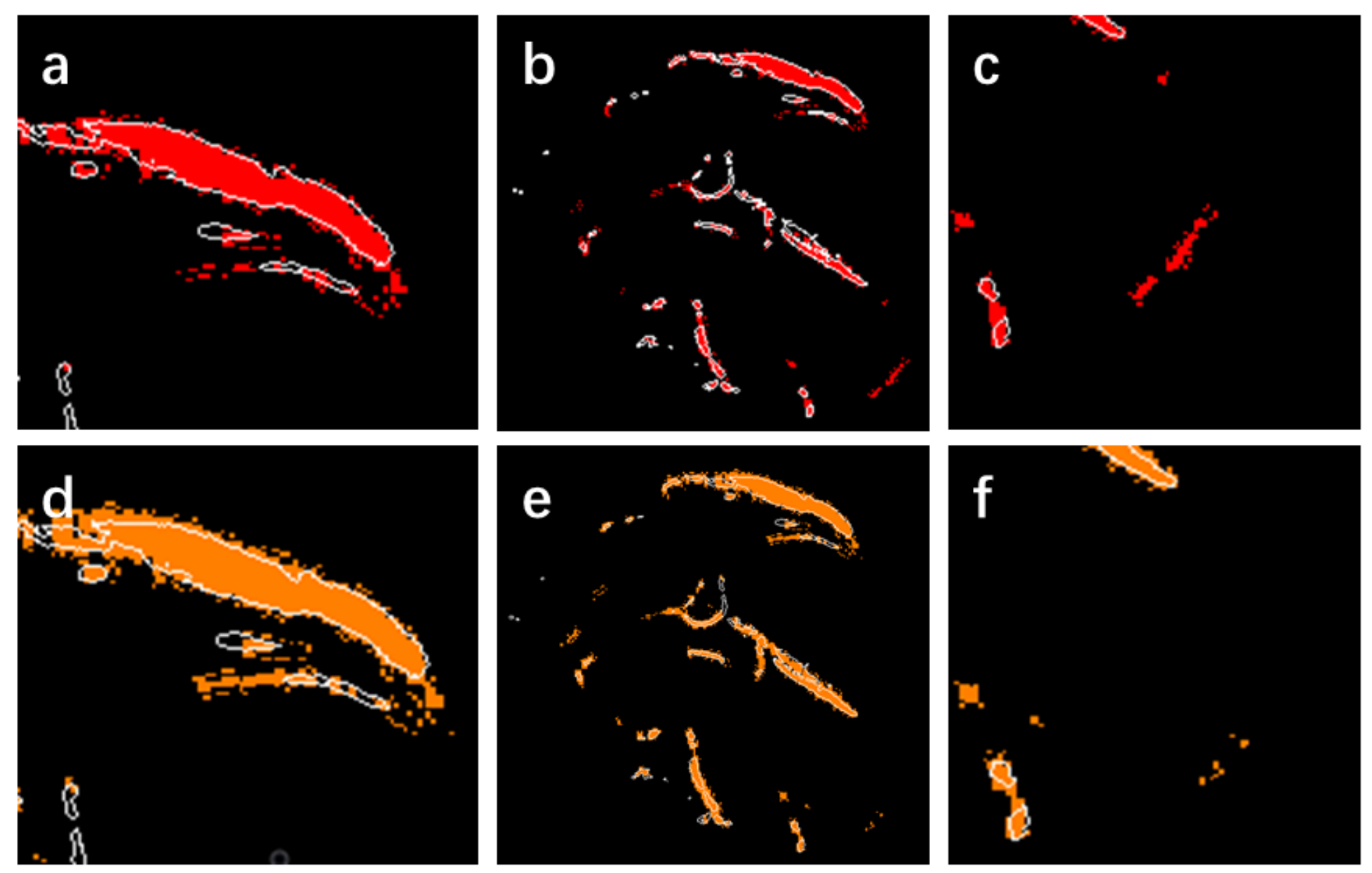

Figure 3

Segmentation details of LBF, and the proposed method. The red areas of $a, b$, and $c$ are the LBF segmentation results, and the yellow areas of $d, e$, and $f$ are the proposed method results. The white outline is ground truth. 


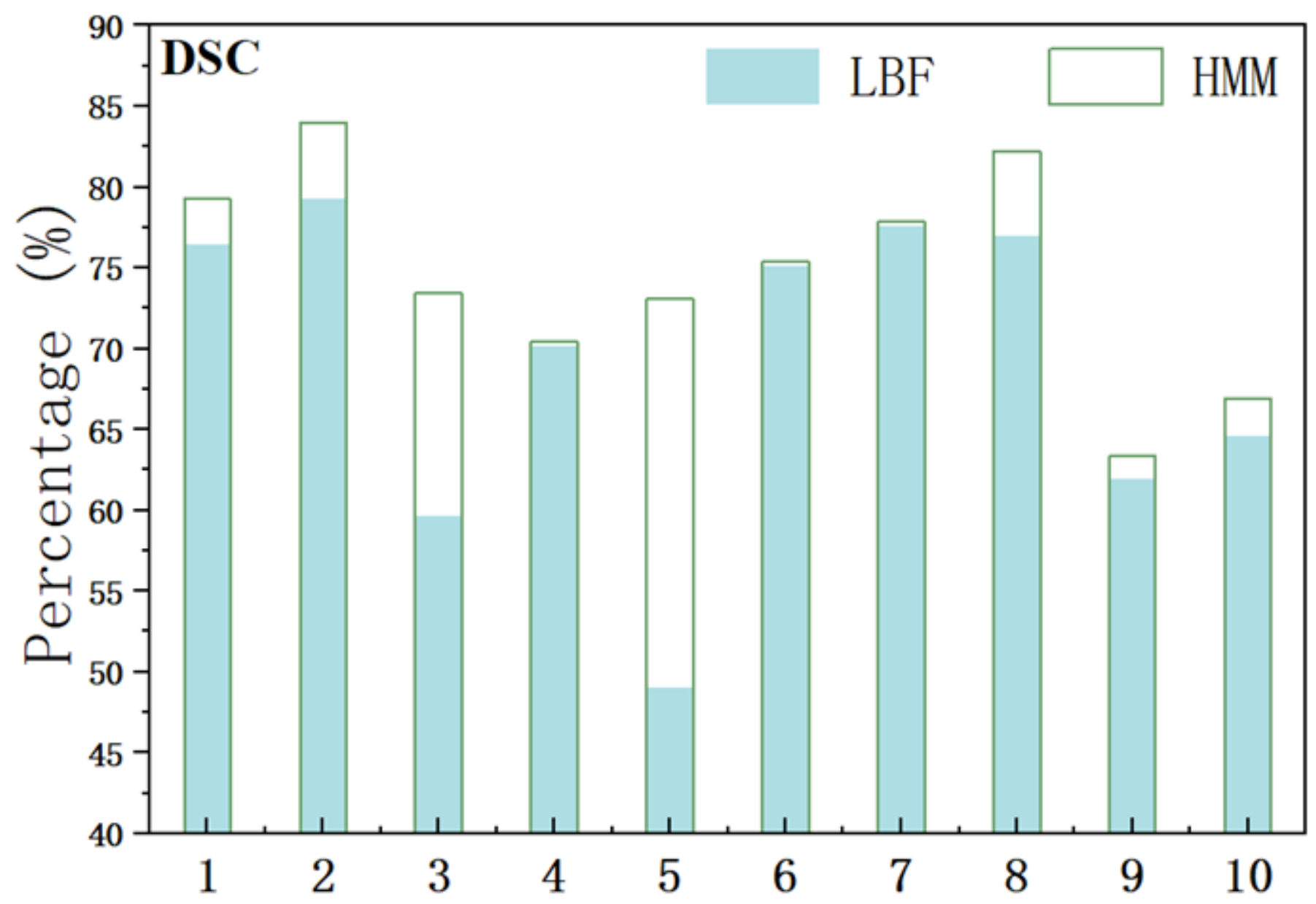

Figure 4

Statistical histogram of DSC. Where, rectangle with a green frame is DSC value of the proposed method. Its blue fills are DSC value of LBF. 


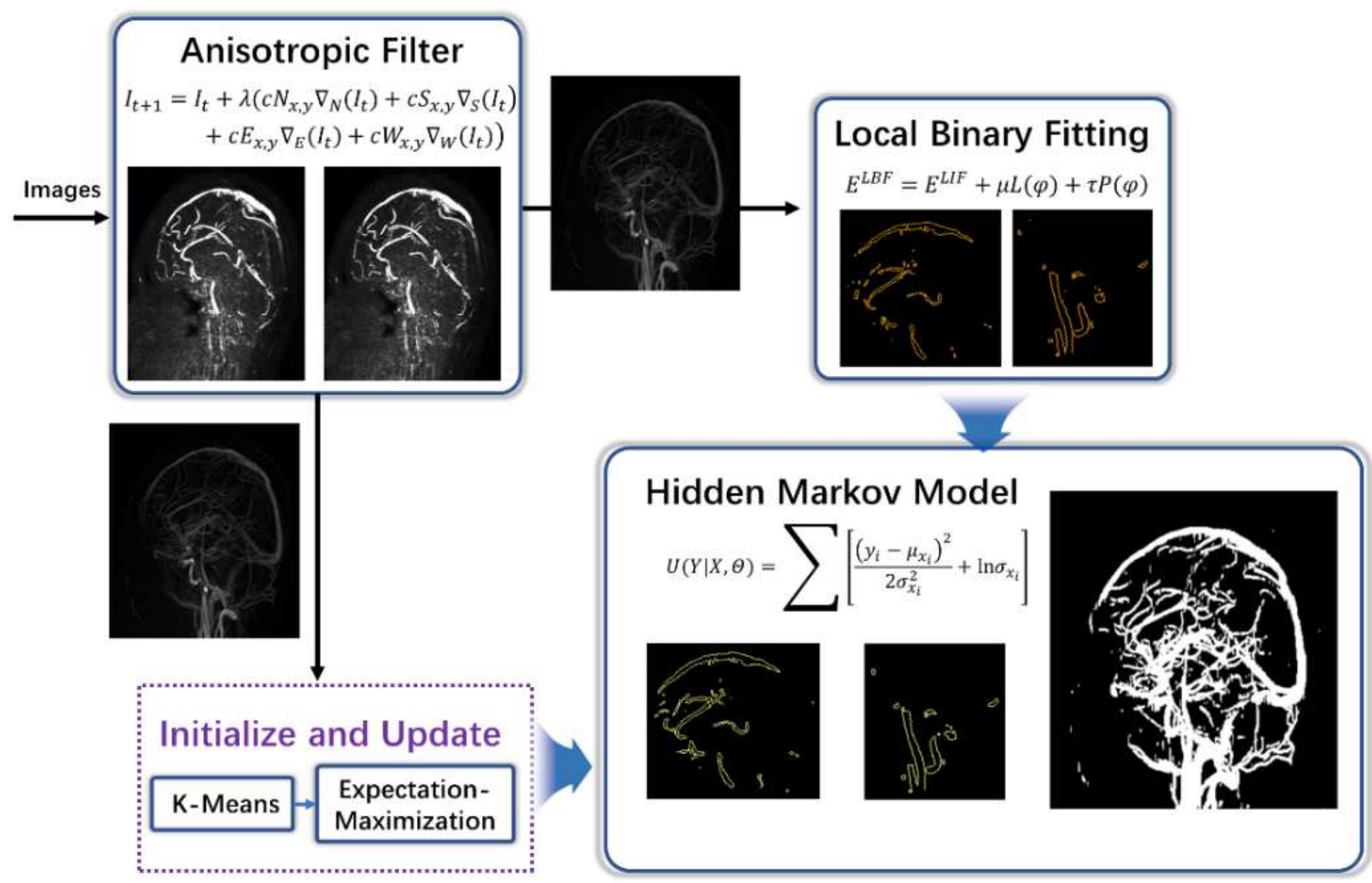

Figure 5

Diagram of the proposed method 

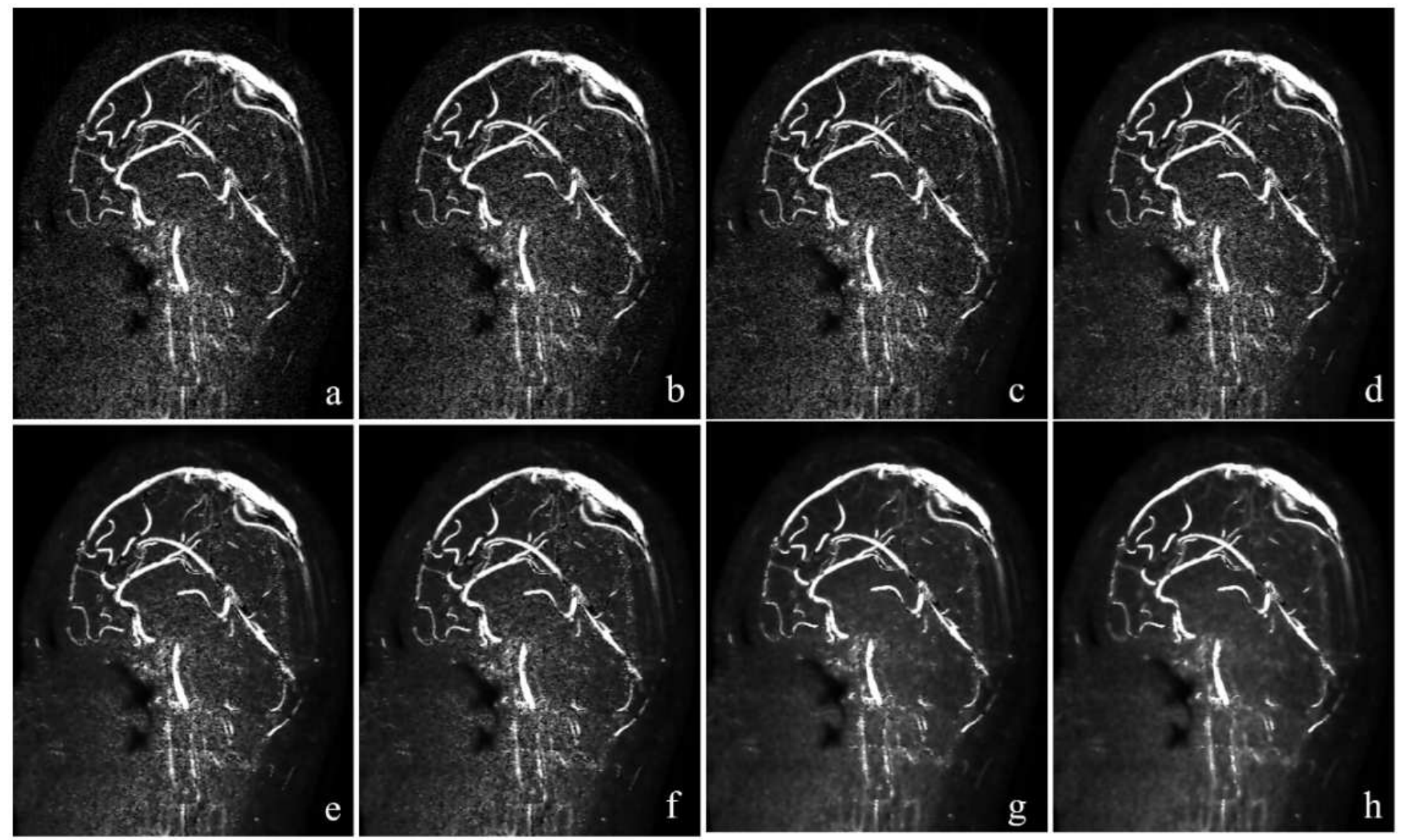

Figure 6

Results of anisotropic filter. a. The original image. $b-\mathrm{h}$. The denoising results when denoising coefficient $\lambda$ is $0.2,0.4,0.6,0.8,1,1.5$, and 2 respectively. 

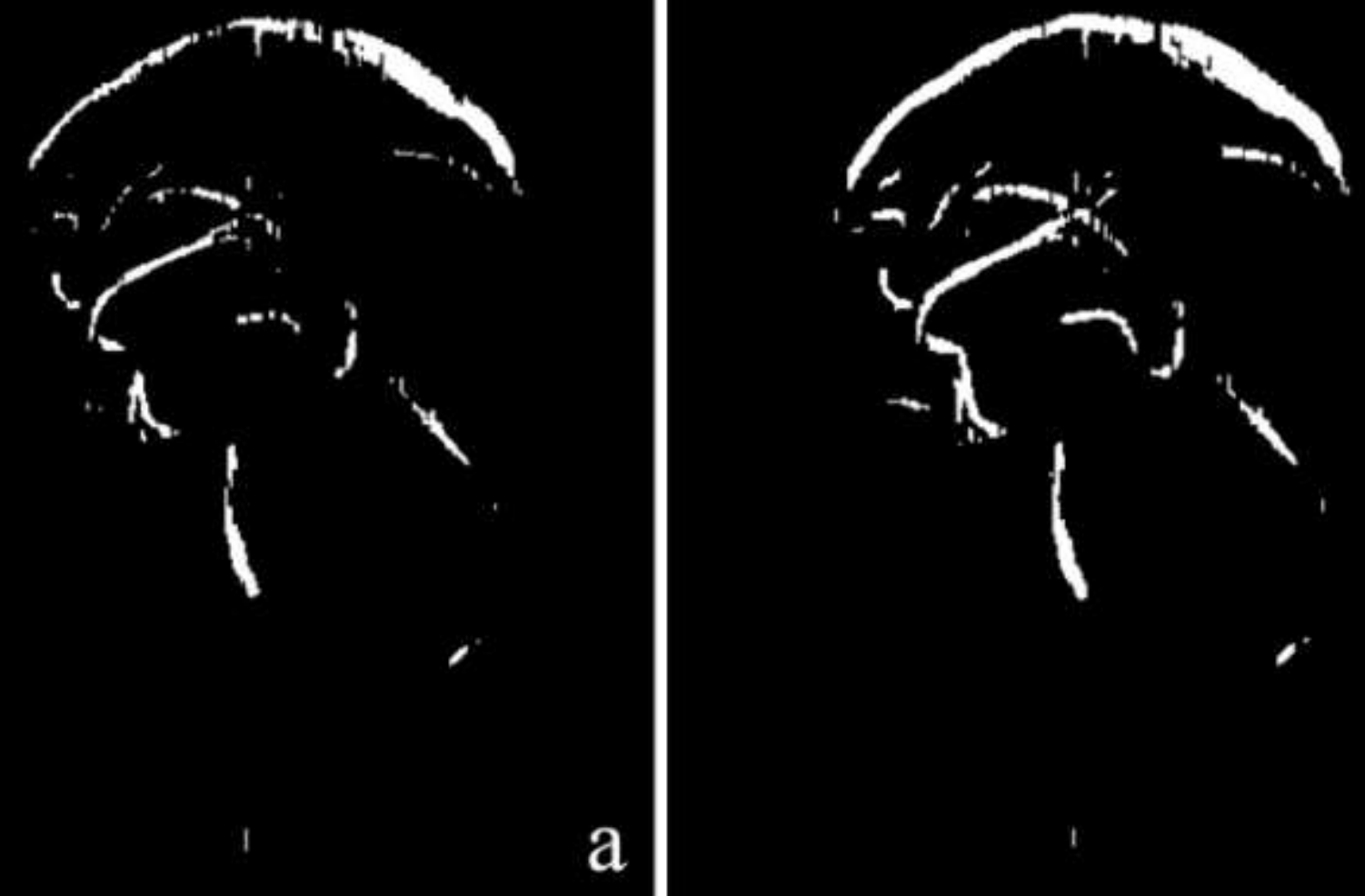

a

Figure 7

Cerebrovascular segmentation results of Figure6f. (a). Result of LBF segmentation. (b). Result of the proposed method. 